This is the peer reviewed version of the following article: Ramos, G. A., CostaCastelló, R., and OIm, J. M. (2015) “Precompensated Second Order Repetitive Control of an Active Filter Under Varying Network Frequency". Asian Journal of Control, 17:1243-1254 which has been published in final form at [doi: 10.1002/asjc.971]. This article may be used for non-commercial purposes in accordance with Wiley Terms and Conditions for Self-Archiving." 


\title{
PRECOMPENSATED SECOND ORDER REPETITIVE CONTROL OF AN ACTIVE FILTER UNDER VARYING NETWORK FREQUENCY
}

\author{
Germán A. Ramos, Ramon Costa-Castelló, and Josep M. Olm
}

\begin{abstract}
Network frequency variations cause a dramatic performance decay in repetitive controller-based shunt active power filters. This problem may be solved by adapting the sampling period in order to keep the ratio between the network period and the sampling period at a constant value. However, these changes may yield closed-loop instability. The introduction of a precompensator that forces the plant to remain invariant despite sampling rate changes allows the use of standard LTI methods in control design and stability analysis as well. Moreover, in order to improve robustness in the face of network frequency estimation uncertainty and sampling time quantization, the regular repetitive controller is replaced by a high order one. Experimental results show the validity of the proposal.
\end{abstract}

\section{INTRODUCTION}

Shunt active power filters are power electronics devices that are connected in parallel with nonlinear and reactive loads. Their main aim is to compensate the disturbances induced by them and, subsequently, to enhance the power quality of the electrical distribution network.

The control of shunt active power filters can be carried out using different approaches $[1,14,11,6,5]$. Most of them are based on two hierarchical control loops: an inner one in charge of assuring the desired network current shape, and an outer one in charge of determining the appropriate power balance. On the one hand, the outer loop displays a slow dynamics and it is usually controlled by means of a PI [7]. On the other hand, the odd-harmonic digital Repetitive Control (RC) method has been successfully used for the inner loop [7]. Nevertheless, this last technique assumes a constant value for the network voltage frequency and undergoes a dramatic performance decay when this value is not properly known or changes with time [20].

This article proposes to overcome the varying network frequency problem with an adaptation of the controller sampling rate according to these changes $[13,12,17]$. However, the use of such a variable sampling rate yields a more involved stability analysis. This is because the inner loop, which answers to a Linear Time Invariant (LTI) model under constant sampling, becomes Linear Time Varying (LTV) when a non-constant sampling is used. This may be addressed with a simple pre-compensation scheme that forces the inner plant to remain invariant despite sampling rate changes; consequently, standard LTI methods can be used in the control design and the stability analysis. This technique, introduced in [16] and validated in a mechatronic plant, is here successfully applied to the shunt active power filter. Notwithstanding, the experimentation in [16] also revealed two other minor, but non negligible, sources of performance decay problems that conventional RC plus the adaptation-compensation scheme could not address: possible uncertainties in the estimation of the network frequency and innacuracy in the implementation of the sampling time due to quantization limitations. However, High Order Repetitive Control (HORC) is well known to efficiently attenuate small mismatches between actual and implemented values of ratio $\frac{T_{n}}{T_{s}}$, where $T_{n}$ and $T_{s}$ stand for the network period and the sampling period of the plant, respectively [20]. Hence, in order to introduce robustness in the face 


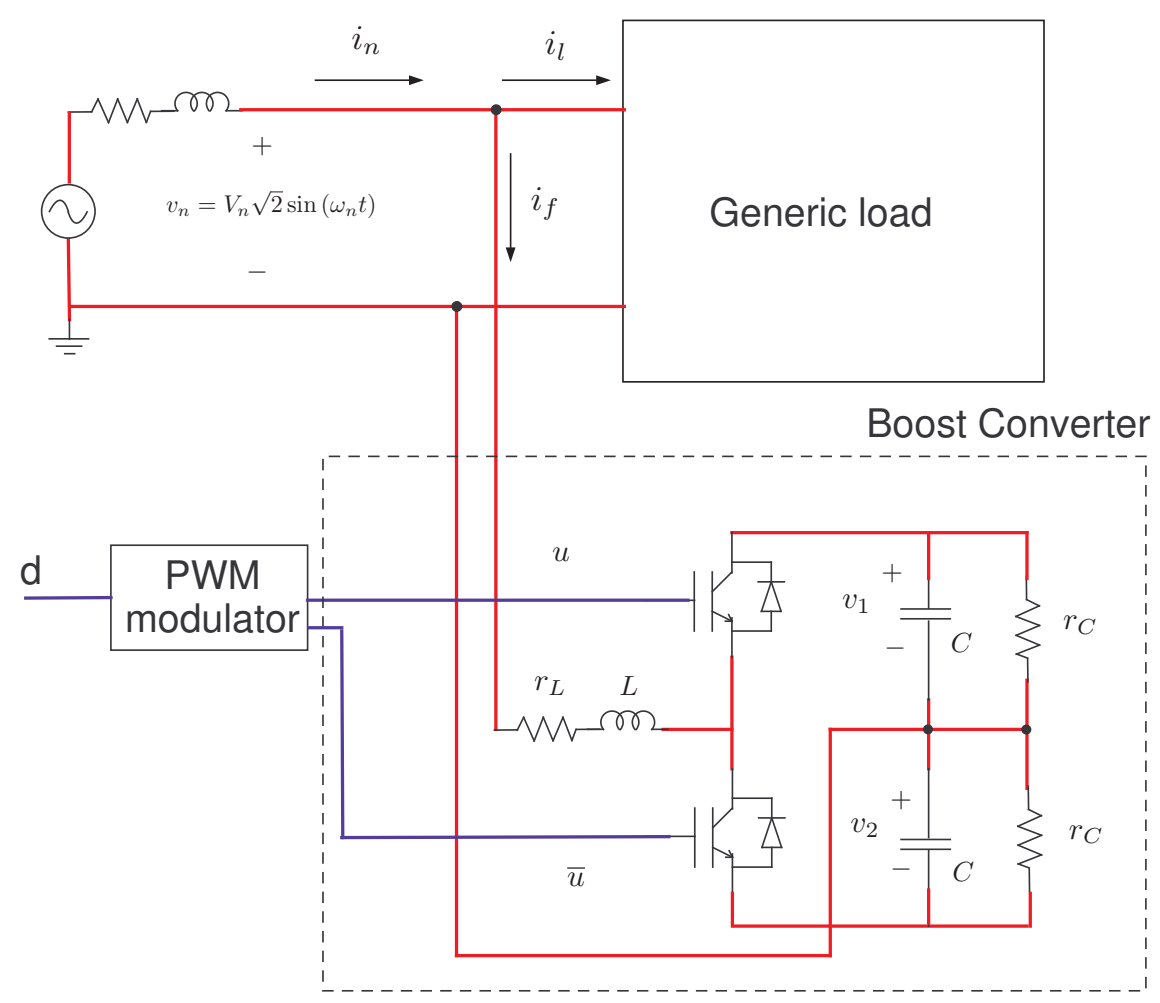

Figure 1: Single-phase shunt active filter.

of such issue, the regular odd-harmonic RC is replaced by an odd-harmonic HORC. Experimental results confirm an excellent performance of the proposed controller.

This article is structured as follows: Section 2 contains the physical plant description and the control goals, as well as a brief description of the overall controller architecture. The proposed control solution is included in Section 3. Experimental results are collected in Section 4 and, finally, conclusions are outlined in Section 5.

\section{ACTIVE FILTER TOPOLOGY AND CONTROLLER ARCHITEC- TURE}

The system architecture is depicted in Figure 1. A boost converter with the ac neutral wire connected directly to the midpoint of the dc bus is used as active filter. The dynamics of the averaged system (at the switching frequency) answers to [7]:

$$
\begin{aligned}
L \frac{d i_{f}}{d t} & =-r_{L} i_{f}+v_{n}-\alpha \\
\frac{d E_{c}}{d t} & =-\frac{2 E_{c}}{r_{C} C}+i_{f} \alpha \\
\frac{d D}{d t} & =-\frac{1}{r_{C} C} D+i_{f}
\end{aligned}
$$

where $\alpha=\frac{1}{2} v_{1}(d+1)+\frac{1}{2} v_{2}(d-1)$, while $E_{c}=\frac{1}{2} C\left(v_{1}^{2}+v_{2}^{2}\right)$ stands for the energy stored in the capacitors, $D=$ $C\left(v_{1}-v_{2}\right)$ stands for the voltage unbalance, $i_{f}$ corresponds to the active filter current, $v_{1}$ and $v_{2}$ are the capacitor voltages, $v_{n}$ stands for network voltage, $L$ is the active filter inductance, and $r_{C}, r_{L}$ represent capacitor and inductor 

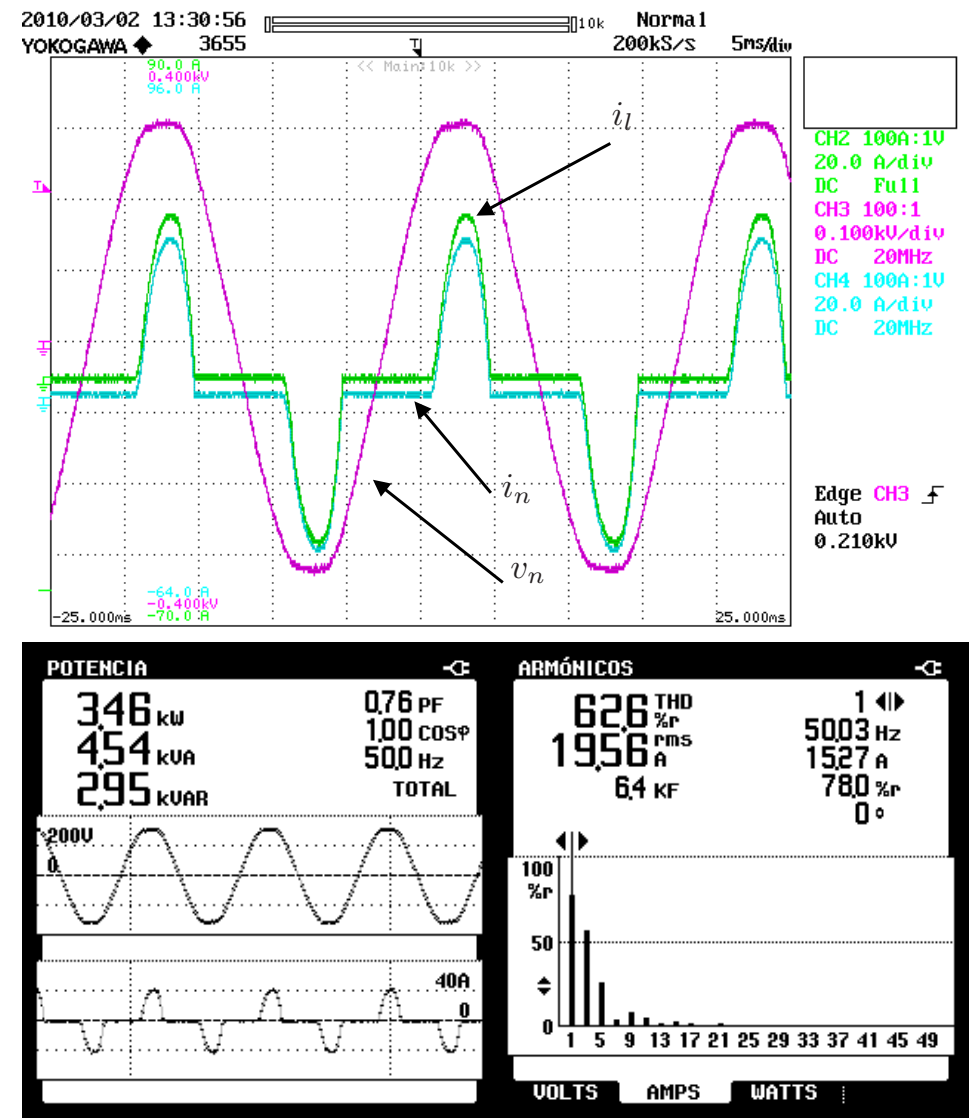

Figure 2: Nonlinear load current and its analysis. (top) $v_{n}, i_{n}$ and $i_{l} v s$ time; (bottom) PF, $\cos \phi$ and THD for $i_{n}$.

parasitic resistances, respectively (see Figure 1). The control variable, $d$, takes its value in the closed real interval $[-1,1]$ and is directly related to the value of the PWM control signal injected to the actual system.

When the active filter is absent and a nonlinear load is plugged to the network, the network current, $i_{n}$, contains harmonic components. This phenomenon is illustrated in Figure 2, where a rectifier is supplying the nonlinear load. However, it should be noticed that $i_{n}$ only contains odd-harmonics: this is a common characteristic of the current generated by most nonlinear loads plugged to the distribution network, and it will be taken into account in the design of the proposed control scheme.

The active filter goal is to assure that the network current is in phase with the voltage source and contains no harmonic components. Hence, denoting $x^{*}$ as the steady-state value of the signal $x(t)$, this goal can be stated as:

$$
i_{n}^{*}=I_{d}^{*} \sin \left(\omega_{n} t\right),
$$

$\omega_{n}=\frac{2 \pi}{T_{n}}$ being the network frequency. Another collateral goal is to assure a constant average value for the dc bus voltage, i.e.

$$
\left\langle v_{1}+v_{2}\right\rangle_{T_{n}}^{*}=v_{d}
$$

where

$$
\langle x\rangle_{T_{n}}=\frac{1}{T_{n}} \int_{t-T_{n}}^{t} x(\tau) d \tau
$$

with $v_{d}>2 \sqrt{2} v_{n}$ (boost condition). Additionally, it is desirable that $v_{1} \approx v_{2}$. 


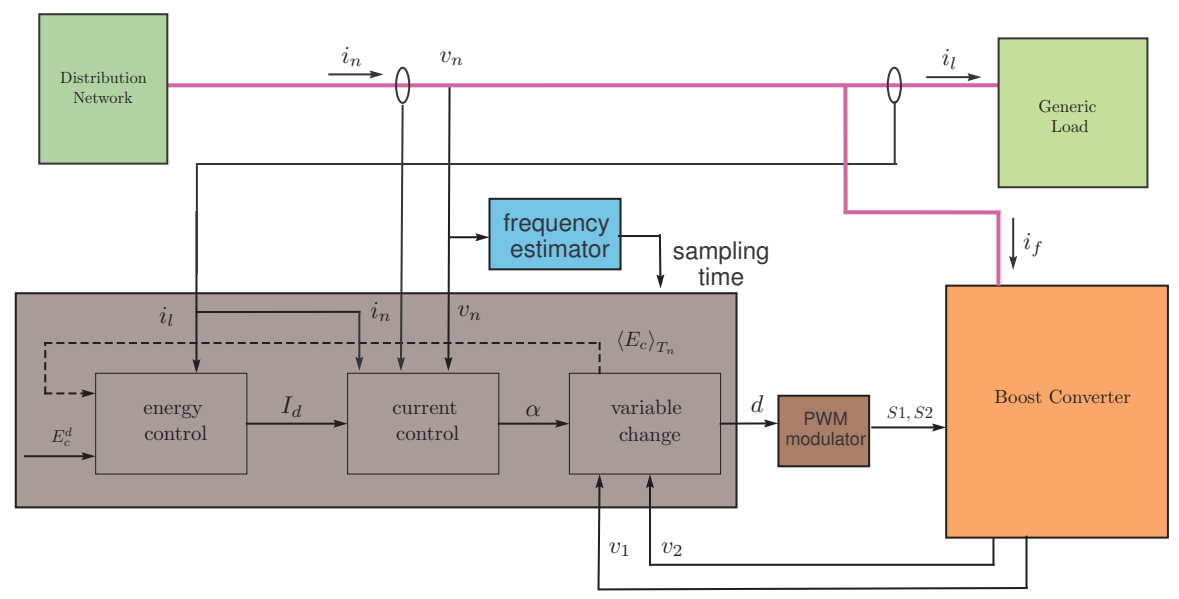

Figure 3: Global architecture of the control system.

Figure 3 shows the hierarchical approach that describes the controller architecture proposed here (see, for example, $[7])$.

Firstly, an RC-based inner current controller forces the sine wave shape by fixing $\alpha$. A new proposal for this controller constitutes the main contribution of this paper, which is presented and analyzed in Section 3.

Secondly, the outer control loop yields the appropriate active power balance for the whole system by fixing the amplitude of the sinusoidal reference, $I_{d}$, for the current control loop. Ideally, $I_{d}$ should be equal to the active component of $i_{l}$, which can be computed in real-time [7]; in practice, $I_{d}$ also must take into account the operation losses. As a consequence, the outer loop is composed of a feedforward term corresponding to the estimated active component of $i_{l}$, and a feedback PI controller in charge of assuring the active power balance. This last feature is achieved if the mean value of the energy stored in the active filter capacitors, $\left\langle E_{C}\right\rangle_{T_{n}}$, is equal to a reference value, namely [7]:

$$
\left\langle E_{C}\right\rangle_{T_{n}}=\left\langle C v_{1}^{2}+C v_{2}^{2}\right\rangle_{T_{n}}=E_{C}^{d} .
$$

The feedforward terms contribute to reduce transient behavior while the feedback action guarantees a correct steadystate behavior.

\section{CURRENT CONTROLLER}

This section contains the proposed control scheme for the current controller. Firstly, Subsection 3.1 introduces oddharmonic RC basics. Then, aiming to compensate for network frequency variations, a time-varying sampling periodbased adaptation-precompensation element is discussed in Subsection 3.2. Then, the lack of robustness to faulty estimations of the network frequency and/or implementations of the sampling period is studied in Subsection 3.3, which motivates the replacement of the odd-harmonic RC by an odd-harmonic HORC introduced in Subsection 3.4.

\subsection{Odd-harmonic repetitive control}

As mentioned in the previous section, the inner control loop is in charge of assuring a sinusoidal current shape. According to the Internal Model Principle (IMP) [4], this is guaranteed by including a sinusoidal generator inside the 
control loop. An IMP-based control technique specifically designed for the tracking/rejection of periodic signals is digital RC $[10,2,15]$.

Digital repetitive controllers are composed of an Internal Model (IM), $G_{i m}(z)$, which assures steady-state performance, and a stabilizing controller, $G_{x}(z)$, which assures closed-loop stability. Traditionally, repetitive controllers are implemented in a "plug-in" fashion, i.e. the repetitive compensator is used to augment an existing nominal controller, $G_{c}(z)$. This nominal compensator is designed to stabilize the plant, $P(z)$, and to provide disturbance attenuation across a broad frequency spectrum. The IM used in RC can be expressed in the following generic form:

$$
G_{i m}(z)=\frac{\sigma W(z) H(z)}{1-\sigma W(z) H(z)},
$$

where $W(z)$ is a delay function, $\sigma$ takes the value 1 or -1 for positive or negative feedback, respectively, and $H(z)$ is a null-phase low-pass FIR filter used to improve robustness in the high frequency range.

The standard IM is obtained from (1) setting $W(z)=z^{-N}, H(z)=1$ and $\sigma=1$ [10]. Notice that $N$ corresponds to the discrete-time period of the signal to be tracked/rejected, namely $N=\frac{T_{n}}{T_{s}}$. With these settings the IM shows full-harmonic action, i.e. it provides tracking/rejection capabilities for any $N$-periodic signal.

As remarked in Section 2, in the active filter case the current only has odd-harmonic components. Hence, an IM that provides action only at odd multiples of the fundamental frequency can be constructed setting $W(z)=z^{-N / 2}$ and $\sigma=-1$ in (1) [8]:

$$
G_{\text {odd }}(z)=\frac{-H(z)}{z^{\frac{N}{2}}+H(z)} .
$$

Such an IM provides faster convergence and simpler implementation [8].

In this work we select $N=\frac{\bar{T}_{n}}{T_{\mathrm{s}}}=400$, which allows a good reconstruction of the continuous-time signals. Hence, assuming a nominal value $\bar{T}_{n}=\frac{1}{50} \mathrm{~s}$, which corresponds to the European network frequency, the nominal sampling rate is set to $\bar{T}_{s}=\frac{1}{20000} \mathrm{~s}$. The overall architecture of the inner control loop is depicted in Figure 4, where A/D and $\mathrm{D} / \mathrm{A}$ stand for analog-to-digital and digital-to-analog converters respectively.

IMs introduce very high gain at harmonic frequencies. Although this is a key feature within the bandwith, it may be problematic at high frequency. In order to preserve high gain within the desired bandwith while introducing robustness in the high frequency range the following low-pass null-phase FIR filter has been used:

$$
H(z)=\frac{1}{4} z^{-1}+\frac{1}{2}+\frac{1}{4} z .
$$

Sufficient stability conditions are established in the following result.

Theorem 1 [8] The closed-loop system depicted in Figure 4 is stable if the following statements hold:

1. The closed-loop system without $R C$, i.e.

$$
T_{o}(z)=\frac{G_{c}(z) P(z)}{1+G_{c}(z) P(z)},
$$

is stable.

2. $\left\|W(z) H(z)\left(1-T_{o}(z) G_{x}(z)\right)\right\|_{\infty}<1$.

Remark 1 These conditions are fulfilled for a proper design of $G_{c}(z), H(z)$ and $G_{x}(z)$. Namely [3, 10]: 


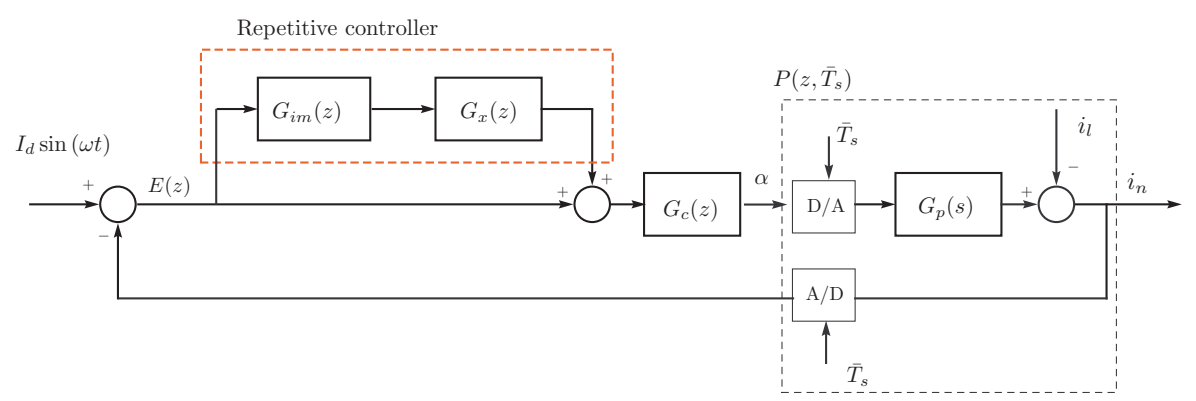

Figure 4: Inner control loop: odd-harmonic repetitive controller.

- The controller $G_{c}(z)$ should provide the system with high enough robustness margin. In this paper we use

$$
G_{c}(z)=-\frac{3.152 z-3.145}{z-0.9985}
$$

- The robustness filter $H(z)$ should be defined to have gain close to 1 within the bandwidth and smaller than 1 outside this bandwidth, which is fulfilled by the one defined in (3).

- The stabilizing filter design, $G_{x}(z)$, is based on phase cancellation techniques; thus, $G_{x}(z)=k_{r}\left(T_{o}(z)\right)^{-1}$ is usually selected in case that $T_{o}(z)$ be minimum phase, while the strategy presented in [21] may be followed for nonminimum phase systems. Notice that there is no problem with the improperness of $G_{x}(z)$ since the IM provides the repetitive controller with a high positive relative degree. Finally, $k_{r}$ can be selected as a trade-off between robustness and transient response, as argued in [9]. Here we use $k_{r}=1$.

The main drawback of RC is its sensitivity to frequency uncertainty [20]. In order to reduce this sensitivity an adaptation-precompesation scheme is proposed in the next subsection.

\subsection{Adaptation-precompesation scheme}

In frameworks where $T_{n}$ is constant, $N$ and $T_{s}$ are designed a priori according to the desired number of samples per period and the technological constraints over $T_{s}$. Unfortunately, the electrical distribution network frequency can undergo fluctuations and, therefore, $T_{n}$ cannot be assumed constant. Furthermore, when the actual ratio $\frac{T_{n}}{T_{s}}$ differs from the implemented value of $N$, the control algorithm performance may decay dramatically [20]. For an active filter, this implies a reduction of the harmonic rejection capabilities and the introduction of reactive current in the system, i.e. a decay of the overall system performance.

The proposed solution is to adapt the plant sampling period $T_{s}$, according to the variation of $T_{n}$, in order to guarantee that the instantaneous values of $\frac{T_{n}}{T_{s}}$ be always equal to $N$. Hence, the network period at the sampling time instant $t_{k}$, i.e. $T_{n}^{k}$, is obtained through a frequency observer, while the corresponding sampling period, $T_{s}^{k}$, is computed using

the relation $T_{s}^{k}=\frac{T_{n}^{k}}{N}$. Nevertheless, this transforms the original LTI system into an LTV one, thus modifying, or even destabilizing, the closed-loop system dynamics. Aiming at annihilating the effect of the time-varying sampling and forcing an output behavior corresponding to that of the nominal sampling period $\bar{T}_{s}$, a precompensator is introduced between the nominal controller $G_{c}(z)$ and the plant [16].

Namely, let the repetitive controller be designed and implemented to provide closed-loop stability for an a priori selected nominal sampling period $\bar{T}_{s}$ (i.e. according to Theorem 1 and Remark 1), using the LTI model (recall Figure 


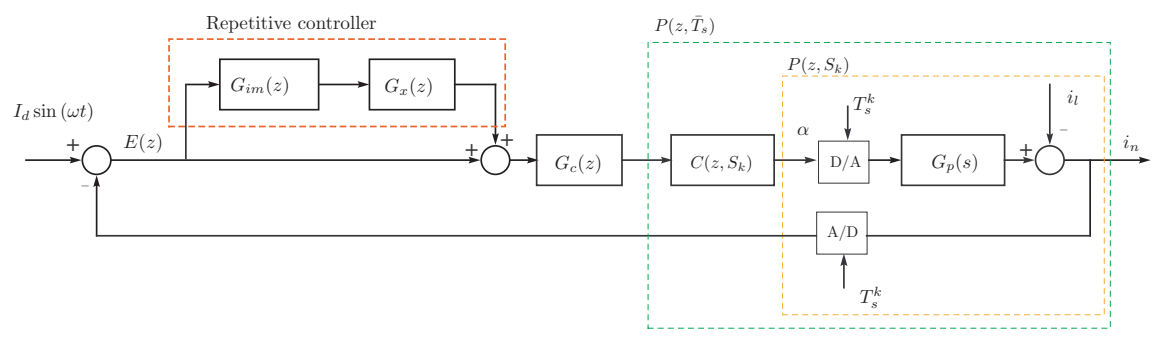

Figure 5: Discrete-time block-diagram of the closed-loop system with the adaptation-precompensation controller structure.

4)

$$
P\left(z, \bar{T}_{s}\right) \triangleq \frac{\operatorname{Num}\left(z, \bar{T}_{s}\right)}{\operatorname{Den}\left(z, \bar{T}_{s}\right)}=\mathfrak{z}\left\{G_{p}(s)\right\},
$$

with

$$
G_{p}(s)=\frac{i_{f}(s)}{\alpha(s)}=-\frac{\frac{1}{r_{L}}}{\left(\frac{L}{r_{L}} s+1\right)(\tau s+1)},
$$

$\tau$ being the time constant of an anti-aliasing filter.

When working at a varying sampling period $T_{s}^{k}$, this model is that of an LTV system:

$$
P\left(z, S_{k}\right)=\frac{\operatorname{Num}\left(z, S_{k}\right)}{\operatorname{Den}\left(z, S_{k}\right)}
$$

with $S_{k}=\left\{T_{s}^{k}, T_{s}^{k-1}, T_{s}^{k-2}\right\}$ (recall that $G_{p}(s)$ is a second order transfer function). It is worth remarking that the use of z-transform notation in an LTV framework is not formally correct; this description is here preserved in order to achieve a compact and simple notation. Hence, $z^{-1}$ should be understood as a one sample time delay, but the sampling interval may change from sample to sample.

On the other hand, in order to annihilate the effect of the sampling rate change, the precompensator

$$
\begin{aligned}
C\left(z, S_{k}\right) & =P\left(z, \bar{T}_{s}\right) P^{-1}\left(z, S_{k}\right)= \\
& =\frac{\operatorname{Num}\left(z, \bar{T}_{s}\right)}{\operatorname{Den}\left(z, \bar{T}_{s}\right)} \cdot \frac{\operatorname{Den}\left(z, S_{k}\right)}{\operatorname{Num}\left(z, S_{k}\right)}
\end{aligned}
$$

is connected in series with the LTV plant $P\left(z, S_{k}\right)$. Thence, the overall behavior is that of the nominal LTI system

$$
C\left(z, S_{k}\right) P\left(z, S_{k}\right)=P\left(z, \bar{T}_{s}\right)
$$

Figure 5 shows the modified inner-loop control scheme.

As the precompensator-plant subsystem is kept invariant and equal to the nominal plant, closed-loop system stability is preserved. Additionally, the inner loop transfer function remains invariant and, as a consequence, outer-loop stability is also preserved. The precompensator (5) can be equivalently implemented in input-output or state-space approaches, but the state-space formulation leads to a more efficient code.

Finally, although this approach guarantees closed-loop stability, it is necessary to check the internal stability of the compensator-plant subsystem $C\left(z, S_{k}\right) P\left(z, S_{k}\right)$, including possible forbidden cancellations. As a result, this analysis will yield a feasible period variation interval $\mathcal{T}$ for which internal stability can be inferred. 


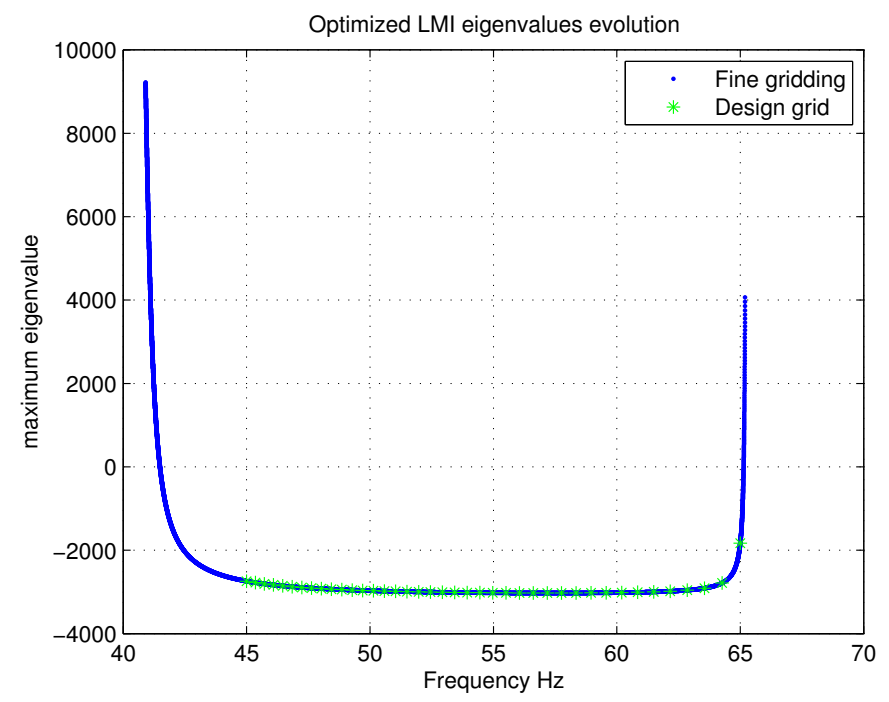

Figure 6: Maximum eigenvalue of $L_{T_{k}}\left(P_{M}\right)$, for $M_{G}$ obtained using $\lambda=100$, a set of 40 homogeneously distributed points and tested with a set of 50000 homogeneously distributed points.

This can be checked using an LMI gridding approach [19]. The method works as follows: let

$$
\begin{aligned}
& x_{k+1}=A_{k} x_{k}+B_{k} u_{k}, \\
& y_{k}=E x_{k}
\end{aligned}
$$

be a realization of $C\left(z, S_{k}\right) P\left(z, S_{k}\right)$, with $E$ being a constant matrix and $A_{k}, B_{k}$ depending continuously on the sampling period $T_{s}$, which takes values in a known, closed interval $\mathcal{T}$; the expressions of $A_{k}, B_{k}, E$ are derived in the Appendix at the end of the paper. In this case, it is well known [18] that if there exist a positive definite symmetric matrix $M$ and a positive real value $\lambda$ such that

$$
L_{T_{s}^{k}}(M)=A_{k}^{\top} M A_{k}-M \leq-\lambda \mathbb{I}, \quad \forall T_{s}^{k} \in \mathcal{T},
$$

then (6) is bounded-input bounded-output stable. Notice that the search of $M$ through (7) results in an infinite set of LMIs, which may be reduced to a finite set using the gridding procedure proposed in [19]. In this way, let $\left\{\tau_{0}, \ldots, \tau_{r}\right\}$ be a sorted set of candidate sampling periods suitably distributed in $\mathcal{T}$. Then, one may solve the following set of LMIs:

$$
L_{\tau_{i}}(M) \leq-\lambda \mathbb{I}, \quad i=0, \ldots, r, \text { s.t. } M=M^{\top}>0,
$$

for a fixed $\lambda \in \mathbb{R}^{+}$. If the problem is feasible and a solution, $M=M_{G}$, is found out, the negative-semidefinite character of $L_{T_{s}^{k}}\left(M_{G}\right)$ has to be checked for intermediate values of $T_{s}^{k}$ in each open subinterval $\left(\tau_{i}, \tau_{i+1}\right)$. If this fails to be accomplished, (8) has to be solved again for a finer grid of $\mathcal{T}$.

Figure 6 shows the maximum eigenvalue of the compensator-plant Lyapunov function derivative, $L_{T_{s}^{k}}(M)$, as a function of the signal frequency $\frac{1}{T_{n}}=\frac{1}{N \cdot T_{s}}$, where $M_{G}$ is found by solving (8) for $\lambda=100$ and a set of 40 equally distributed values of $T_{s}^{k}$ in $\mathcal{T}=\left[\frac{1}{45 \cdot N}, \frac{1}{65 \cdot N}\right]$. It can be seen that it is negative for a wide range of values of $T_{s}$. As a consequence, $T_{s}$ may vary within a very important range of periods covering the most relevant frequency interval from the practical point of view, namely $[45,65] \mathrm{Hz}$. 


\subsection{Robustness analysis}

As mentioned in Subsection 3.1, any fault in the estimation of $T_{n}$ and/or implementation of $T_{s}$ causes a difference between the experimental ratio $\frac{T_{n}}{T_{s}}$ and the implemented value for $N$, which degrades the repetitive control performance. This phenomenon is analysed in this section using the sensitivity function.

The sensitivity transfer function of the closed-loop system in Figure 4, obtained using the generic IM (1), is

$$
S(z)=\frac{E(z)}{R(z)}=S_{o}(z) S_{M o d}(z),
$$

where

$$
S_{o}(z)=\frac{1}{1+G_{c}(z) P(z)}
$$

stands for the sensitivity function of the system without repetitive controller and $S_{M o d}(z)$ is the modifying sensitivity function

$$
S_{M o d}(z)=\frac{1-\sigma W(z) H(z)}{1-\sigma W(z) H(z)\left(1-G_{x}(z) T_{o}(z)\right)} .
$$

As an example, the blue line in Figure 7 shows the first harmonic magnitude response evolution of the modifying sensitivity function against a relative deviation of the real sampling period $\hat{T}_{s}^{k}$ with respect to the "nominal" $T_{s}^{k}$, namely

$$
\left|S_{M o d}\left(e^{\frac{2 \pi j}{N\left(1+\Delta T_{S}\right)}}\right)\right|
$$

where $S_{M o d}(\cdot)$ is defined in (10) using $\sigma=-1, H(z)=1, G_{x}(z)=1 / T_{o}(z)$, and

$$
\Delta T_{s}=\frac{\hat{T}_{s}^{k}-T_{s}^{k}}{T_{s}^{k}} .
$$

Notice that even small deviations of $\hat{T}_{s}^{k}$ entail an important performance reduction for odd-harmonic RC [16], a situation which is even worst at higher harmonics. This behavior may be importantly alleviated by HORC, as explained in the next subsection.

It is worth noticing that, since invariance has already been established, closed-loop stability is not threatened unless $\hat{T}_{s}^{k} \notin \mathcal{T}$.

\subsection{Odd-harmonic high-order repetitive control}

It was shown in the previous subsection that performance in conventional RC may be affected by network frequency estimation errors and/or sampling period quantization problems. This paper suggests to replace the RC introduced in Section 3.1 by a second order odd-harmonic RC which, as displayed in Figure 7, enhances robustness against frequency uncertainty by flattening the peak of the modifying sensitivity function. Hence, the adaptation-precompensation scheme introduced in Section 3.2 will be in charge of providing robustness in the face of large variations of $T_{n}$, while odd-harmonic HORC will provide robustness in the face of estimation and quantization errors.

The main difference between regular RC and HORC is that the latter uses a weighted sum of delays instead of a single delay. Specifically, an $M$-th order odd-harmonic RC employs the delay function

$$
W(z)=\sum_{k=1}^{M} w_{k} z^{-k N / 2} .
$$




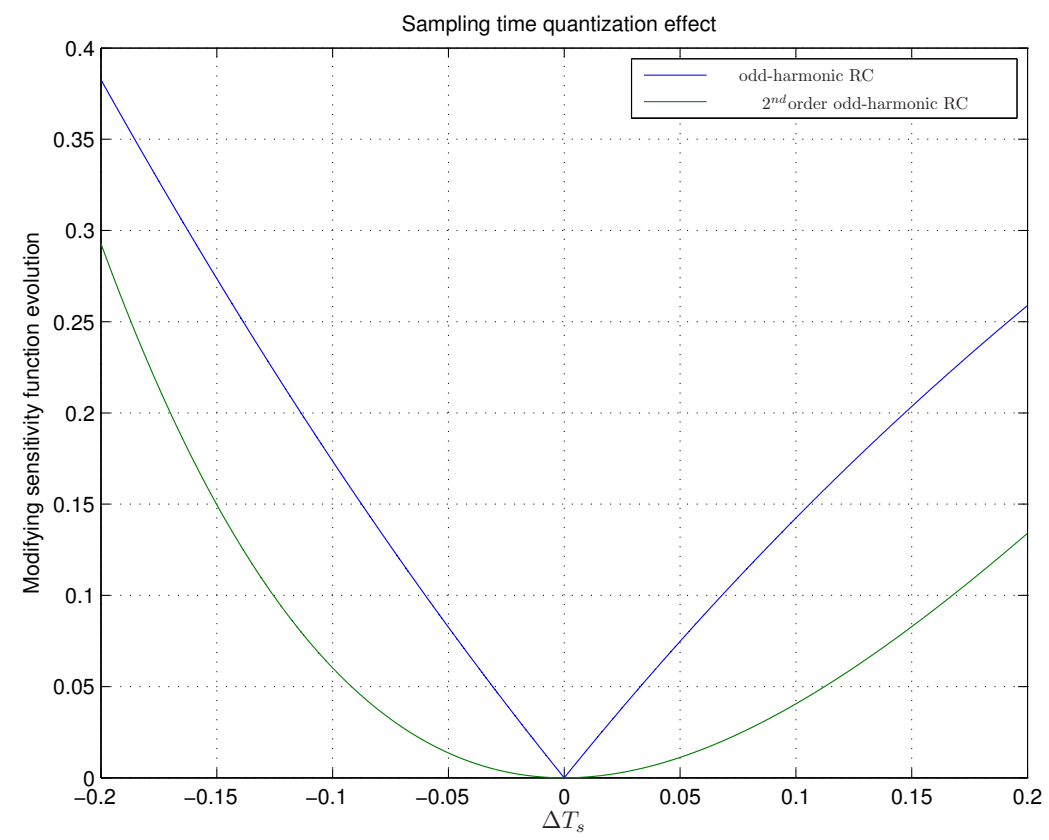

Figure 7: First harmonic modifying sensitivity function evolution over sampling time implementation error.

This follows the delay structure suggested in [20] for a full-harmonic HORC. The weights $w_{k}$ are derived in order to obtain period variation robustness [20] using the maximally flat concept. This is achieved demanding infinite gain for the $\operatorname{IM}(1)$ with $H(z)=1, \sigma=-1$, at the odd-harmonic frequencies, i.e.

$$
W\left(e^{j \omega_{k}}\right)=-1, \quad \omega_{k}=\frac{2(2 k-1) \pi}{N},
$$

where $k=1,2,3, \ldots, N / 2$, while its first $M-1$ derivatives at these frequencies are set to 0 . This yields the following linear system for the weights:

$$
\sum_{k=1}^{M}(-1)^{k} k^{p} w_{k}=\left\{\begin{array}{lll}
-1 & \text { if } & p=0 \\
0 & \text { if } & p=1, \ldots, M-1 .
\end{array}\right.
$$

Hence, for a second order odd-harmonic IM one has that $M=2, w_{1}=2$ and $w_{2}=1$, which yields

$$
G_{\text {hodd }}(z)=-\frac{\left(2 z^{-\frac{N}{2}}+z^{-N}\right) H(z)}{1+\left(2 z^{-\frac{N}{2}}+z^{-N}\right) H(z)} .
$$

This IM allows small deviations in the implemented sampling period without losing the original RC performance features.

The stability conditions introduced in Section 3.1 also hold for HORC, while the adaptation-precompensation scheme introduced in Section 3.2 guarantees plant invariance. Consequently, the overall closed-loop system does not require any additional stabilizing elements or re-tuning stages. 


\section{EXPERIMENTAL RESULTS}

The experimental setup is composed of a full-bridge diode rectifier acting as a nonlinear load, the previously described single-phase active filter with $L=1 \mathrm{mH}, r_{L}=0.5 \Omega, C=9.9 \mathrm{mF}, r_{C}=8200 \Omega, \tau=0.03568 \mathrm{~ms}$, and a PACIFIC Smartsource (140-AMX-UPC12) that acts as a variable frequency ac power source.

The active filter controller has been implemented on a DSP based hardware, i.e. within a digital framework, with a nominal sampling frequency, $\bar{T}_{s}^{-1}$, equal to the switching frequency of $20 \mathrm{kHz}$. The actual network period $T_{n}$ is obtained from the network voltage zero crossings through some additional hardware and a digital low-pass filter that runs in the DSP. Due to the combined effect of sampling rate adaptation and HORC, frequency observer precision is not a critical deal. With this information, the sampling period $T_{s}$ is updated in real time to maintain the ratio $N=400$.
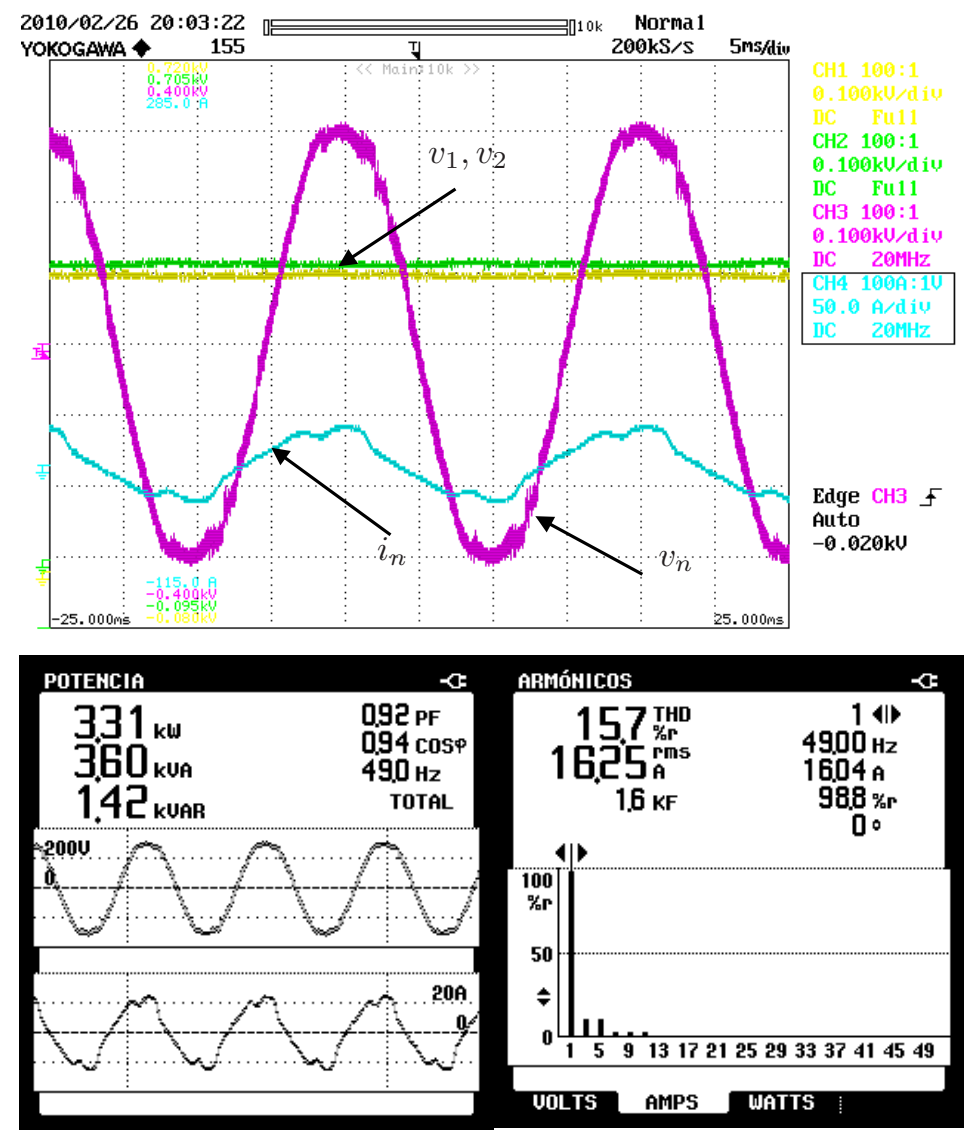

Figure 8: Steady-state active filter behaviour under nonlinear load conditions at $49 \mathrm{~Hz}$ using conventional RC with constant sampling time. (top) $v_{n}, i_{n}, v_{1}$ and $v_{2} v s$ time; (bottom) PF, $\cos \phi$ and THD for $i_{n}$.

Figures 8 and 9 show the steady-state performance when using RC and HORC with constant sampling time, i.e. without the adaptive pre-compensation scheme, and the frequency is deviated to $49 \mathrm{~Hz}$. It can be seen that, in both cases, the current signal at the source, $i_{n}$, is not completely sinusoidal since it contains small additional harmonic components. Thus, the THD is $15.7 \%$ and $4.6 \%$ for RC and HORC, respectively, while cos $\phi$ and the Power Factor 

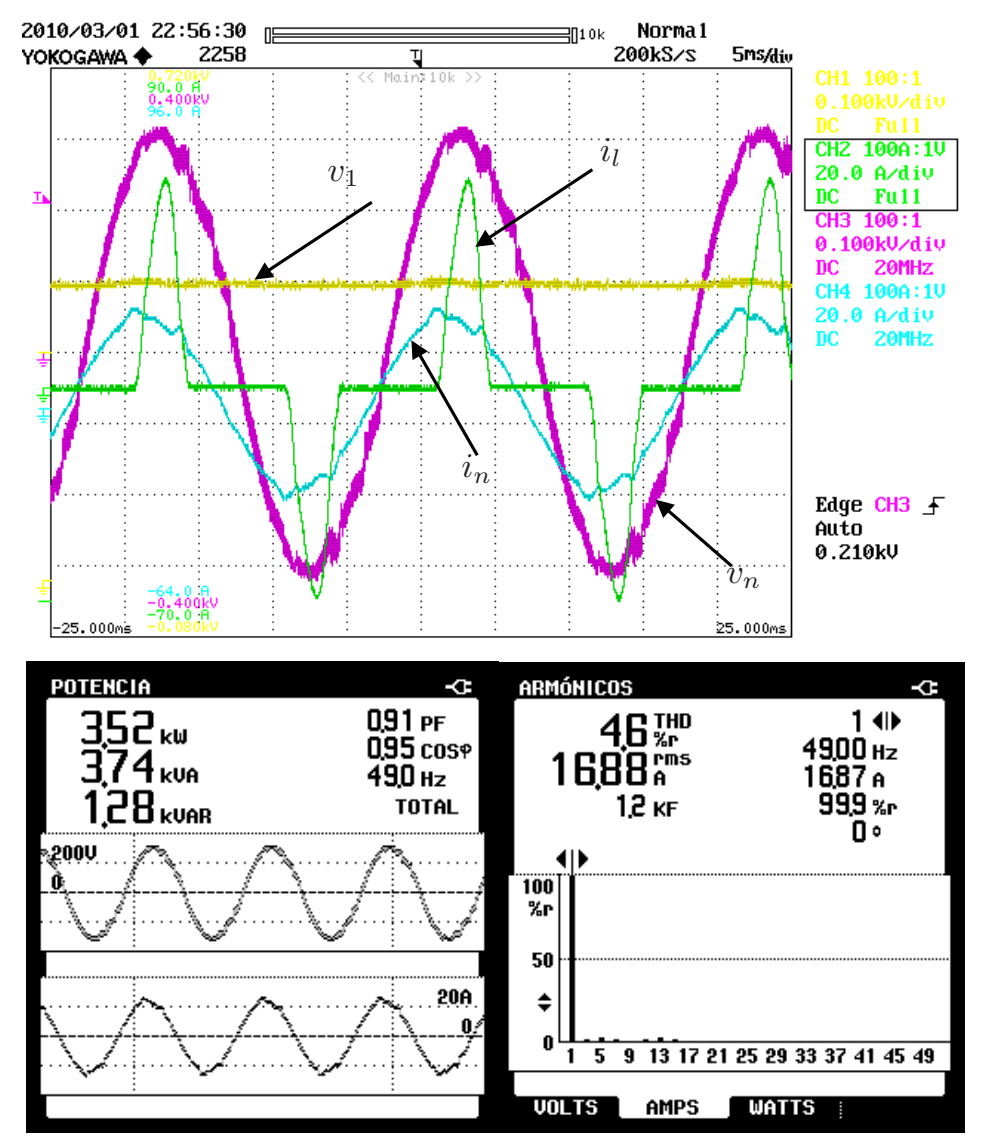

Figure 9: Steady-state active filter behaviour under nonlinear load conditions at $49 \mathrm{~Hz}$ using HORC with constant sampling time. (top) $v_{n}, i_{n}, i_{l}$ and $v_{1} v s$ time; (bottom) PF, $\cos \phi$ and THD for $i_{n}$.

(PF) take values between 0.92 and 0.95 . Furthermore, the obtained results show that, as expected, HORC yields lower performance degradation compared to conventional RC.

A remarkable improvement is obtained when the adaptation-precompensation scheme is combined with a second order odd-harmonic RC. Figures 10 and 11 show the steady-state behavior at $48 \mathrm{~Hz}$ and $52 \mathrm{~Hz}$, respectively: the shape of the source current is nearly sinusoidal with a THD of $0.4 \%$, while PF and $\cos \phi$ at the port are unitary.

Finally, good transient behaviors are illustrated in Figures 12, 13 and 14. Specifically, Figures 12 and 13 depict the transients when the load is switched off and on, respectively; in both cases the capacitor voltages are maintained close to the reference value -though only $v_{1}$ is shown due to hardware limitations- and the system reaches the steady state in a few cycles. In turn, Figure 14 shows the system behavior when the source frequency changes from $48 \mathrm{~Hz}$ to $50 \mathrm{~Hz}$ in a 20 cycles ramp manner. Notice that the required sinusoidal shape of the network current is preserved, while the variable network voltage frequency is adapted. The capacitor voltage $v_{1}$ is maintained almost constant; $v_{2}$ behaves in a similar manner, but it is not shown due to the above exposed reason. 


\section{CONCLUSIONS}

This work has presented a digital controller for an active filter application based on RC. The architecture of the control system, its stability analysis and some of its design issues have been described. The controller includes an adaptation pre-compensation scheme to follow possible network frequency variations without losing the advantages of RC and maintaining its low computational cost. This technique assures inner-loop invariance and, consequently, outer-loop stability, independent from sampling period variations and network frequency observer dynamics. Moreover, a second order odd-harmonic repetitive controller makes the system robust to uncertainty in the network frequency estimation and in the sampling period quantization.

In summary, the proposed scheme preserves the RC performance and, at the same time, is insensitive to network frequency variations and sampling period implementation mismatches.

\section{References}

[1] S. Buso, L. Malesani, and P. Mattavelli. Comparison of current control techniques for active filters applications. IEEE Trans. Ind. Electron., 45(5):722-729, 1998.

[2] K. Chew and M. Tomizuka. Digital control of repetitive errors in disk drive systems. IEEE Control Syst. Magazine, pages 16-19, 1990.

[3] R. Costa-Castelló, J. Nebot, and R. Griñó. Demonstration of the internal model principle by digital repetitive control of an educational laboratory plant. IEEE Trans. Education, 48(1):73-80, 2005.

[4] B. Francis and W. Wonham. Internal model principle in control theory. Automatica, 12(5):457-465, 1976.

[5] F. Freijedo, J. Doval-Gandoy, O. Lopez, P. Fernandez-Comesana, and C. Martinez-Penalver. A signal-processing adaptive algorithm for selective current harmonic cancellation in active power filters. IEEE Trans. Ind. Electron., 56(8):2829-2840, 2009.

[6] H. Fujita. A single-phase active filter using an h-bridge pwm converter with a sampling frequency quadruple of the switching frequency. IEEE Trans. Power Electron., 24(4):934 -941, 2009.

[7] R. Griñó, R. Cardoner, R. Costa-Castelló, and E. Fossas. Digital repetitive control of a three-phase four-wire shunt active filter. IEEE Trans. Ind. Electron., 54(3):1495-1503, 2007.

[8] R. Griñó and R. Costa-Castelló. Digital repetitive plug-in controller for odd-harmonic periodic references and disturbances. Automatica, 41(1):153-157, 2005.

[9] G. Hillerström and R. C. Lee. Trade-offs in repetitive control. Technical Report CUED/F-INFENG/TR 294, University of Cambridge, 1997.

[10] T. Inoue, M. Nakano, T. Kubo, S. Matsumoto, and H. Baba. High accuracy control of a proton synchroton magnet power supply. In Proc. $8^{\text {th }}$ IFAC World Congress, pages 216-220, 1981.

[11] C. Lascu, L. Asiminoaei, I. Boldea, and F. Blaabjerg. Frequency response analysis of current controllers for selective harmonic compensation in active power filters. IEEE Trans. Ind. Electron., 56(2):337 -347, 2009.

[12] J. Liu and Y. Yang. Frequency adaptive control technique for rejecting periodic runout. Control Eng. Practice, 12(1):31-40, 2004.

[13] T. J. Manayathara, T. Tsao, and J. Bentsman. Rejection of unknown periodic load disturbances in continous steel casting process using learning repetitive control approach. IEEE Trans. Cont. Syst. Tech., 4(3):259-265, 1996. 
[14] P. Mattavelli. A closed-loop selective harmonic compensation for active filters. IEEE Trans. Ind. Applicat., 37(1):81-89, 2001.

[15] J. Na, R. Costa-Castelló, R. Griñó, and X. Ren. Discrete-time repetitive controller for time-delay systems with disturbance observer. Asian Journal of Control, 14(5):1340-1354, 2012.

[16] J. M. Olm, G. A. Ramos, and R. Costa-Castelló. Adaptive compensation strategy for the tracking/rejection of signals with time-varying frequency in digital repetitive control systems. J. Process Control, 20(4):551-558, 2010.

[17] J. M. Olm, G. A. Ramos, and R. Costa-Castelló. Odd-harmonic repetitive control of an active filter under varying network frequency: Control design and stability analysis. In Proc. American Control Conference, pages $1749-1754,2010$.

[18] W. Rugh. Linear system theory, 2nd Ed. Prentice-Hall, Inc., Upper Saddle River, NJ, 1996.

[19] A. Sala. Computer control under time-varying sampling period: An LMI gridding approach. Automatica, 41(12):2077-2082, 2005.

[20] M. Steinbuch. Repetitive control for systems with uncertain period-time. Automatica, 38(12):2103-2109, 2002.

[21] M. Tomizuka, T. Tsao, and K. Chew. Analysis and synthesis of discrete-time repetitive controllers. Journal of Dynamic Systems, Measurement, and Control, 111(3):353-358, 1989.

\section{APPENDIX}

The realization of the compensator-plant subsystem given in (6) is derived as follows. A state-space Jordan form for the transfer function of the active filter system plus the anti-aliasing filter (4), is given by:

$$
\begin{aligned}
\dot{x}_{p}(t) & =A_{c p} x(t)+B_{c p} u(t) \\
y_{p}(t) & =E_{p} x_{p}(t),
\end{aligned}
$$

with

$$
A_{c p}=\left[\begin{array}{cc}
-\frac{r_{L}}{L} & 1 \\
0 & -\frac{1}{\tau}
\end{array}\right] B_{c p}=\left[\begin{array}{l}
0 \\
1
\end{array}\right] E_{p}^{\top}=\left[\begin{array}{c}
-\frac{1}{L \tau} \\
0
\end{array}\right] .
$$

The discretization of the (13)-(14) for a certain $T_{s}^{k}$ is

$$
\begin{aligned}
x_{k+1}^{p} & =A_{p}\left(T_{s}^{k}\right) x_{k}^{p}+B_{p}\left(T_{s}^{k}\right) u_{k} \\
y_{k}^{p} & =E_{p} x_{k}^{p},
\end{aligned}
$$

where

$$
A_{p}\left(T_{s}^{k}\right):=\exp \left(A_{c p} T_{s}^{k}\right), B_{p}\left(T_{s}^{k}\right):=\int_{0}^{T_{s}^{k}} e^{A_{c p} s} d s B_{c p}
$$

while for a nominal sampling period $\bar{T}_{s}$ it boils down to

$$
\begin{aligned}
\bar{x}_{k+1}^{p} & =A_{p}\left(\bar{T}_{s}\right) \bar{x}_{k}^{p}+B_{p}\left(\bar{T}_{s}\right) \bar{u}_{k} \\
\bar{y}_{k}^{p} & =E_{p} \bar{x}_{k}^{p} .
\end{aligned}
$$

The control law $u_{k}$ such that $y_{k}=\bar{y}_{k}$ is:

$$
u_{k}=D_{k}\left(A_{p}\left(\bar{T}_{s}\right) \bar{x}_{k}^{p}-A_{p}\left(T_{s}^{k}\right) x_{k}^{p}+B_{p}\left(\bar{T}_{s}\right) \bar{u}_{k}\right),
$$


where $D_{k}=D_{k}\left(T_{s}^{k}\right)=\left(E_{p} B_{p}\left(T_{s}^{k}\right)\right)^{-1} E_{p}$; in this case $\left(E_{p} B_{p}\left(T_{s}^{k}\right)\right)^{-1}$ always exists. However, since the state of the plant is not available, the following LTV model is used to obtain an estimation $\tilde{x}_{k}^{p}$ of the state $x_{k}^{p}$ :

$$
\tilde{x}_{k+1}^{p}=A_{p}\left(T_{s}^{k}\right) \tilde{x}_{k}^{p}+B_{p}\left(T_{s}^{k}\right) u_{k} .
$$

Hence, in the realization (6) one has that:

$$
\begin{gathered}
x=\left[\begin{array}{c}
\bar{x}^{p} \\
\tilde{x}^{p} \\
x^{p}
\end{array}\right], B_{k}=\left[\begin{array}{c}
B\left(\bar{T}_{s}\right) \\
F_{k} B\left(\bar{T}_{s}\right) \\
F_{k} B\left(\bar{T}_{s}\right)
\end{array}\right], E=\left[\begin{array}{c}
0 \\
0 \\
E_{p}
\end{array}\right], \\
A_{k}=\left[\begin{array}{ccc}
A_{p}\left(\bar{T}_{s}\right) & 0 & 0 \\
F_{k} A_{p}\left(\bar{T}_{s}\right) & \left(\mathbb{I}-F_{k}\right) A_{p}\left(T_{s}^{k}\right) & 0 \\
F_{k} A_{p}\left(\bar{T}_{s}\right) & -F_{k} A_{p}\left(T_{s}^{k}\right) & A_{p}\left(T_{s}^{k}\right)
\end{array}\right],
\end{gathered}
$$

with $F_{k}=F_{k}\left(T_{s}^{k}\right)=B_{p}\left(T_{s}^{k}\right) D_{k}\left(T_{s}^{k}\right)$. 

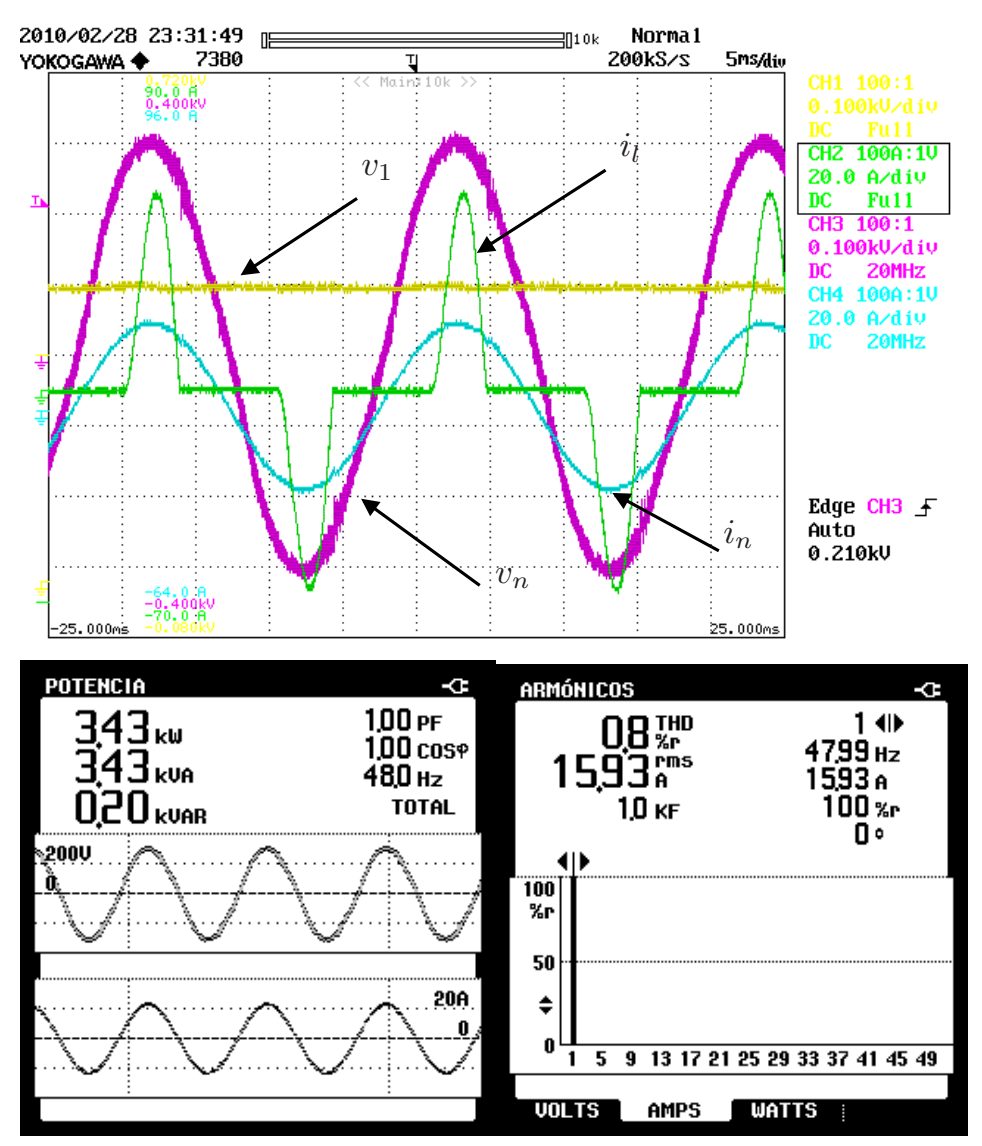

Figure 10: Steady-state active filter behaviour under nonlinear load conditions at $48 \mathrm{~Hz}$ using the proposed controller architecture. (top) $v_{n}, i_{n}, i_{l}$ and $v_{1} v s$ time; (bottom) PF, $\cos \phi$ and THD for $i_{n}$.
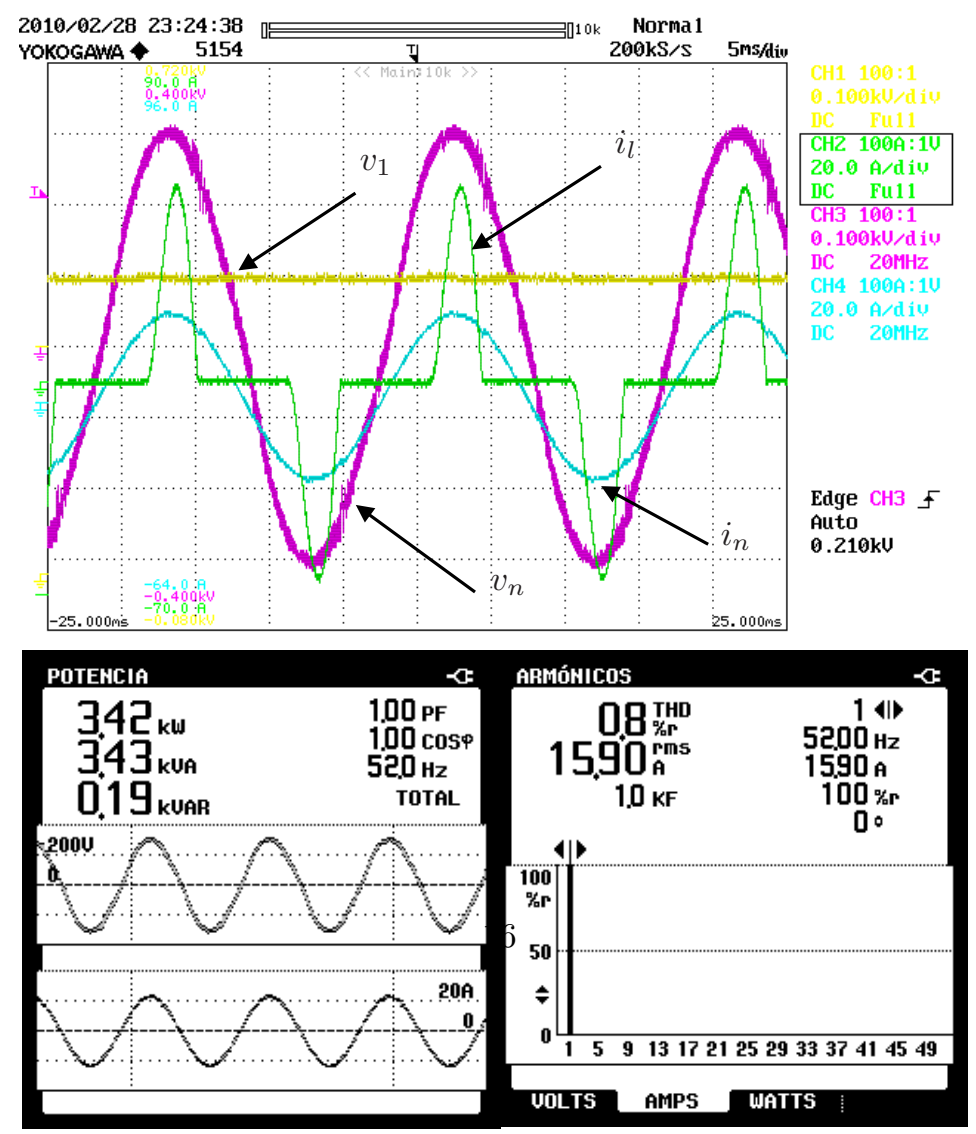


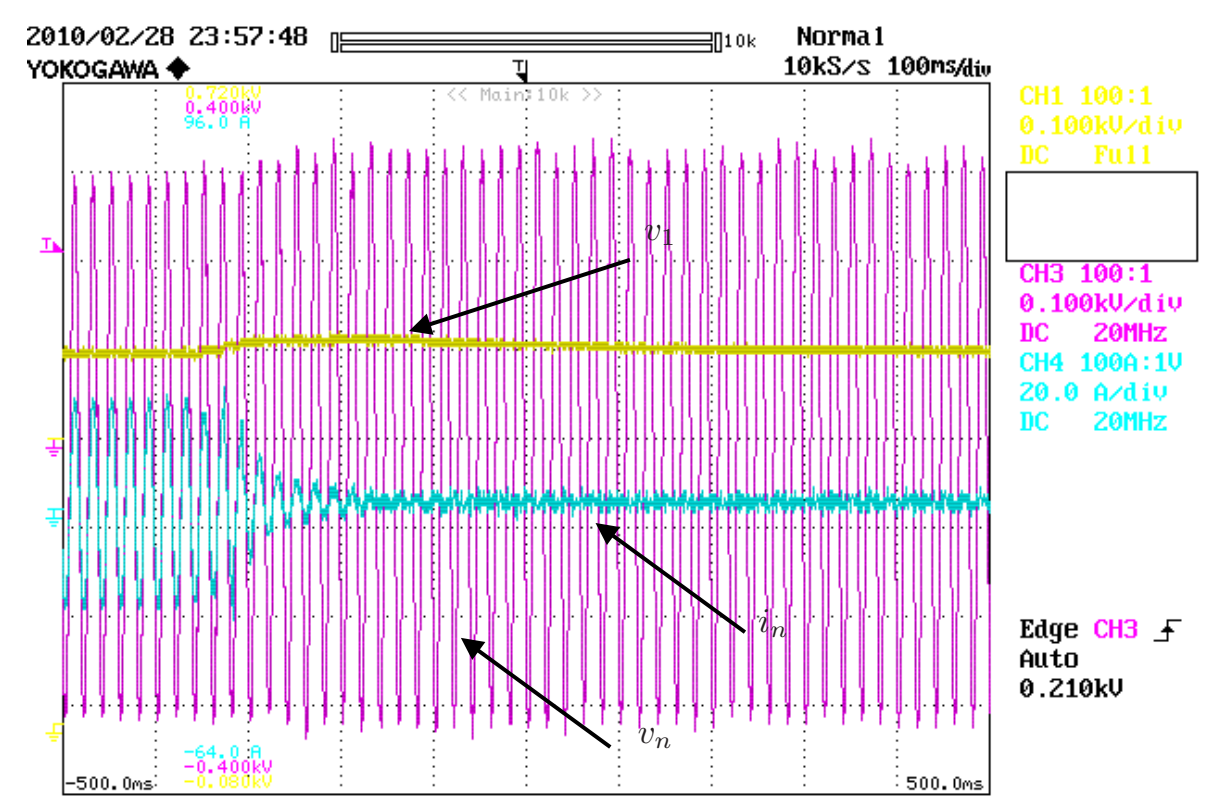

Figure 12: Active filter transient behaviour : switching off loads. $v_{n}, i_{n}, i_{l}$ and $v_{1} v s$ time;

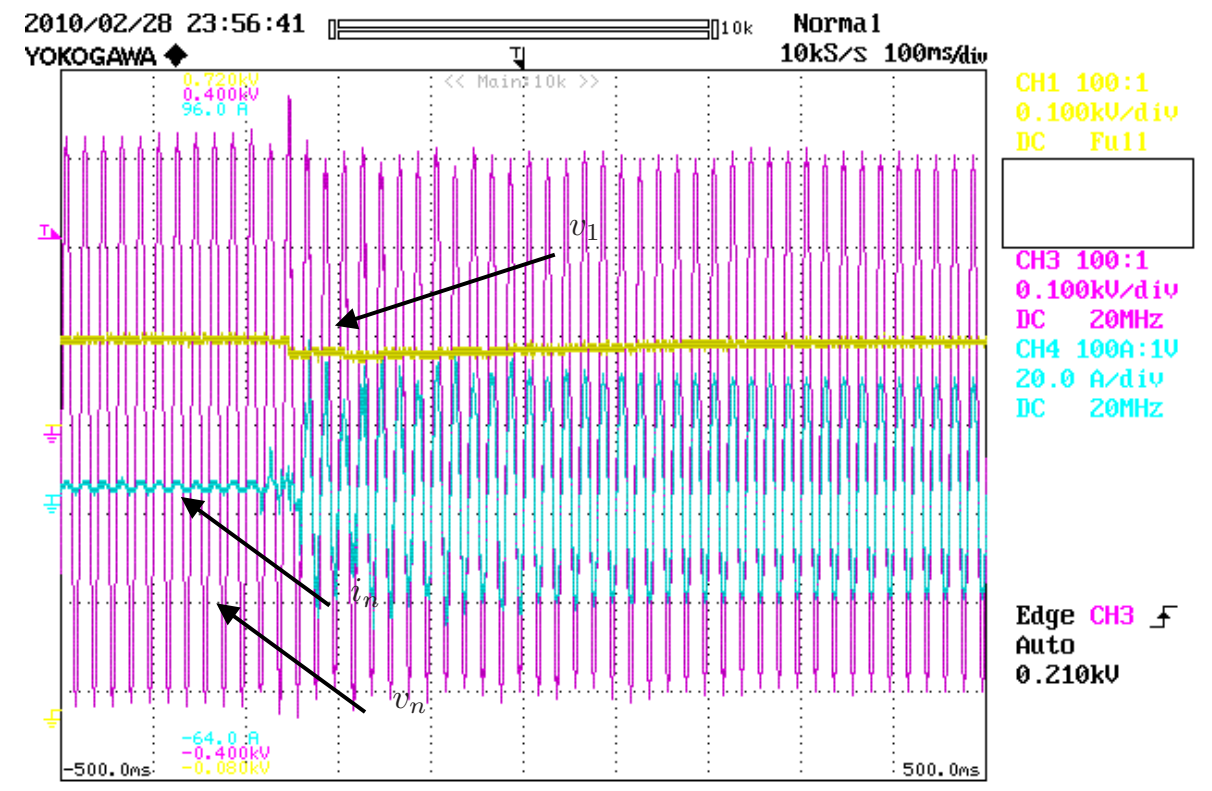

Figure 13: Active filter transient behaviour : switching on loads. $v_{n}, i_{n}, i_{l}$ and $v_{1} v s$ time;. 


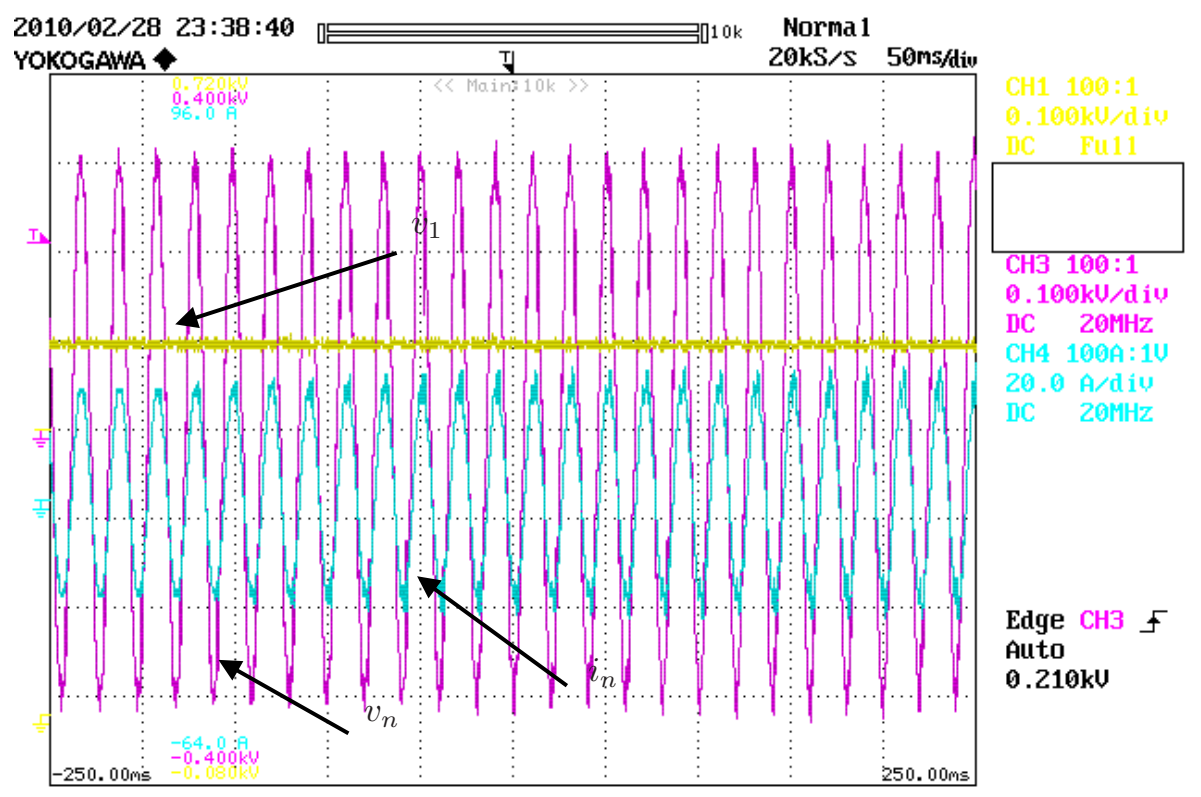

Figure 14: Active filter transient behaviour : frequency changes following a ramp from $48 \mathrm{~Hz}$ to $52 \mathrm{~Hz}$ in 40 cycles. $v_{n}$, $i_{n}, i_{l}$ and $v_{1}$ vs time;. 